\title{
Two novel bocaparvovirus species identified in wild Himalayan marmots
}

\author{
Yuanyun $\mathrm{Ao}^{1 \dagger}$, Xiaoyue $\mathrm{Li}^{2 \dagger}$, Lili $\mathrm{Li}^{1}$, Xiaolu Xie ${ }^{3}$, Dong $\mathrm{Jin}^{4}$, Jiemei $\mathrm{Yu}^{1^{*}}$, Shan $\mathrm{Lu}^{4^{*}}$ \& \\ Zhaojun Duan ${ }^{1 *}$ \\ ${ }^{1}$ National Institute for Viral Diseases Control and Prevention, Chinese Center for Disease Control and Prevention, Beijing 100052, China; \\ ${ }^{2}$ Laboratory Department, the First People's Hospital of Anqing, Anqing 246000, China; \\ ${ }^{3}$ Peking Union Medical College Hospital, Beijing 100730, China; \\ ${ }^{4}$ National Institute for Communicable Disease Control and Prevention, Chinese Center for Disease Control and Prevention, \\ Beijing 102206, China
}

Received September 10, 2017; accepted September 16, 2017; published online December 1, 2017

\begin{abstract}
Bocaparvovirus (BOV) is a genetically diverse group of DNA viruses and a possible cause of respiratory, enteric, and neurological diseases in humans and animals. Here, two highly divergent BOVs (tentatively named as Himalayan marmot BOV, HMBOV1 and HMBOV2) were identified in the livers and feces of wild Himalayan marmots in China, by viral metagenomic analysis. Five of 300 liver samples from Himalayan marmots were positive for HMBOV1 and five of 99 fecal samples from these animals for HMBOV2. Their nearly complete genome sequences are 4,672 and 4,887 nucleotides long, respectively, with a standard genomic organization and containing protein-coding motifs typical for BOVs. Based on their NS1, NP1, and VP1, HMBOV1 and HMBOV2 are most closely related to porcine BOV SX/1-2 (approximately 77.0\%/50.0\%, 50.0\%/53.0\%, and $79.0 \% / 54.0 \%$ amino acid identity, respectively). Phylogenetic analysis of these three proteins showed that HMBOV1 and HMBOV2 formed two distinctly independent branches in BOVs. According to these results, HMBOV1 and HMBOV2 are two different novel species in the Bocaparvovirus genus. Their identification expands our knowledge of the genetic diversity and evolution of BOVs. Further studies are needed to investigate their potential pathogenicity and their impact on Himalayan marmots and humans.
\end{abstract}

emerging infectious diseases, bocaparvovirus, novel, Himalayan marmot

Citation: $\quad$ Ao, Y., Li, X., Li, L., Xie, X., Jin, D., Lu, S., Yu, J., and Duan, Z. (2017), Two novel bocaparvovirus species identified in wild Himalayan marmots. Sci China Life Sci, 60, 1348-1356, https://doi.org/10.1007/s11427-017-9231-4

\section{INTRODUCTION}

Recently, many severe, emerging infectious diseases have occurred in humans after the respective viruses overcame the inter-species barriers. Indeed, $60 \%-80 \%$ of human infectious diseases are estimated to have originated in wild animals (Jones et al., 2008). Recently emerging viruses, including

$\dagger$ Contributed equally to this work

*Corresponding authors (Jiemei Yu, email: yjm1982@126.com; Zhaojun Duan, email: zhaojund@126.com; Shan Lu, email: lushan@icdc.cn)
Severe Acute Respiratory Syndrome (SARS), Middle East Respiratory Syndrome (MERS), and the Ebola virus (Drosten et al., 2003; Haagmans et al., 2014; Gire et al., 2014), are the most well-known examples, which have caused a large number of human deaths and high economic losses. The discovery of novel viruses in wild animals is the first step in predicting their potential threat to human and animal health. The early detection and characterization of viruses infecting wild animals may therefore allow the prediction of their threat to human health.

Parvoviridae comprises a group of small, non-enveloped, 
single-stranded DNA viruses that can infect a wide range of animals and insects. According to the classification system of the International Committee on Taxonomy of Viruses (ICTV) (http://ictvonline.org/virusTaxonomy.asp), parvoviruses can be subdivided into two subfamilies: Parvovirinae and Densovirina. The sub-family Parvovirinae that infects mammals, currently consists of the genera Protoparvovirus, Erythroparvovirus, Aveparvovirus, Copiparvovirus, Dependoparvovirus, Amdoparvovirus, Tetraparvovirus, and Bocaparvovirus. These viruses can cause severe diseases in humans and animals, including fifth disease in humans (HPV B19) (Balkhy et al., 1998) and stillbirth, mummification, embryonic death, and infertility in pigs (PPV) (Christianson et al., 1992).

Bocaparvovirus (BOV), a member of the Parvoviridae family, has a genome of approximately 5.0 kilobases, including an open reading frame (ORF), NP1, located between the genes encoding NS1 and VP1/VP2 (Manteufel and Truyen, 2008). BOVs are most commonly associated with respiratory and gastrointestinal symptoms in young humans and animals (Manteufel and Truyen, 2008; Jartti et al., 2012), affecting both public health and economic development. For example, human bocaparvovirus (HBOV1) and porcine bocaparvovirus 1 (PBOV1) are associated with respiratory symptoms (Rotzén-Östlund et al., 2014; Jula et al., 2013), and HBoV2-4, gorilla BOV, and PBOV with diarrhea and acute flaccid paralysis (Cashman and O'Shea, 2012; Kapoor et al., 2009; Kapoor et al., 2010; Meng, 2012; Gunn et al., 2015 ). BOVs have also been identified in cerebrospinal fluid (Yu et al., 2013), liver (Li et al., 2013), lung (Cságola et al., 2012), and blood (Zhai et al., 2010), although there is no strong evidence supporting the association of their diseases. Since the initial identification in canine and bovine stool samples (Abixanti et al., 1961; Binn et al., 1970), BOVs have been detected in many mammalian species, including humans, pigs (Cheng et al., 2010), cows (Yang et al., 2016), California sea lions (Li et al., 2011), cats (Lau et al., 2012), gorillas (Kapoor et al., 2009), rabbits (Lanave et al., 2015), and bats (He et al., 2013). BOVs have a high mutation rate and undergo frequent recombination events, similar to those of RNA viruses (Shackelton et al., 2005; Hoelzer et al., 2008). Thus, the discovery of novel BOVs and new animal hosts will expand our knowledge of their genetic diversity and evolution. It will also provide a better understanding of the potential of these viruses for cross-species transmission and emergence.

Viral metagenomics based on high-throughput sequencing technology has proved to be a powerful technique for identifying novel viruses, and thus an important tool in diagnostic virology and viral disease prevention and control. In China, wild marmots (Marmota himalayana), whose habitat is the
Qinghai-Tibetan Plateau, 2,800-4,000 m above sea level, are the primary animal reservoir of plague. As yet, the viruses in wild marmots have rarely been reported. However, with the expansion of human activities, such as human domesticating marmots as pets, hunting them for meat and fur and using them as viral animal model (hepatitis B virus), as well as choosing Qinghai-Tibetan Plateau for travel, the rapid spread of viruses between humans and Marmota himalayana is becoming possible. As a result, it is very important to conduct a thorough survey of viruses in Himalayan marmots to understand their basic viral epidemiological data. Using viral metagenomics analysis, we discovered a novel hepatitis A virus and diverse astrovirus species in fecal and liver samples from Himalayan marmots, as previously reported (Yu et al., 2016; Ao et al., 2017).

Here, we report the discovery, also in this viral metagenomics, of two novel BOVs in liver and fecal samples obtained from Himalayan marmots in the Qinghai-Tibetan Plateau, China. In addition, we describe the nearly complete sequences and detailed genomic organization of these two BOVs (tentatively named as Himalayan marmot BOV, HMBOV1 and HMBOV2). Genomic and phylogenetic analyses indicated that they comprise different novel species of BOV. Screening for HMBOV1 and HMBOV2 was also carried out in 300 liver and 99 fecal samples from Himalayan marmots in China.

\section{RESULTS}

\section{Identification of novel BOV sequences}

In a BLASTX analysis, 14 reads from two pools of liver samples and 43 reads from two pools of fecal samples had amino acid sequence similarity to BOVs, based on a customized informatics pipeline with minor modifications. Assembly of the unique reads from the liver and fecal samples generated five contigs (approximately $250 \mathrm{bp}$ ) and eight contigs (250-1524 bp), respectively. The low identities with the sequences of porcine BOVs suggested the presence of novel BOVs. Thus, one liver and one fecal sample positive for the BOVs HMBOV1 and HMBOV2 were selected for viral genome amplification and sequencing.

\section{Prevalence of HMBOV1 and HMBOV2}

Five of the 300 liver samples from the Himalayan marmots were positive for HMBOV1 and showed $99.0 \%$ nucleotide identity with each other. Reverse transcriptase heminested PCR showed that five of the 99 fecal samples were positive for HMBOV2, displaying 100.0\% nucleotide identity (Figure 1A). However, HMBOV1 was not detected in any of the stool samples nor was HMBOV2 identified in any of the liver samples. 

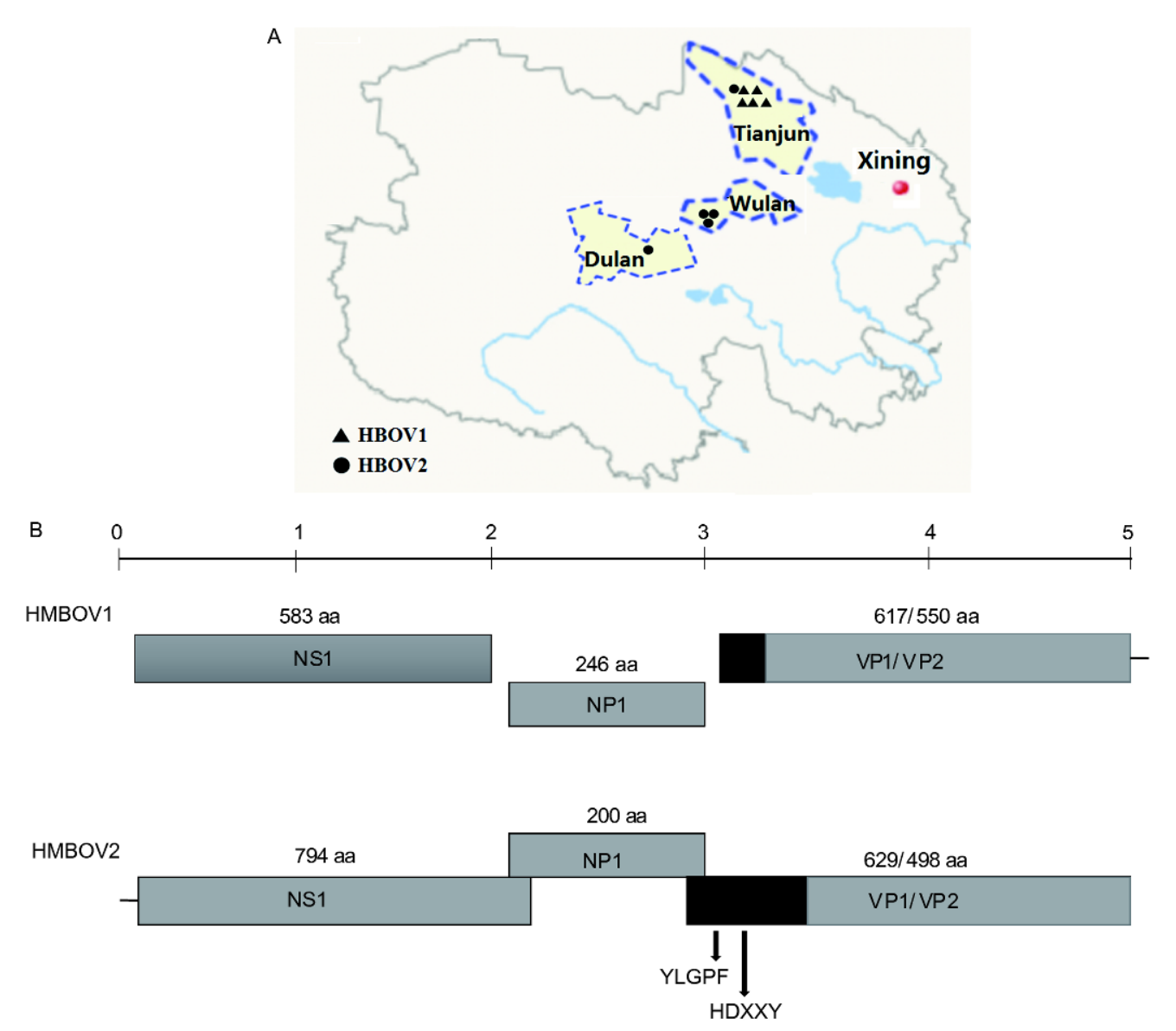

Figure 1 (Color online) The distribution and genomes of novel bocaparvoviruses. A, Geographic distribution of bocaparvoviruses in Himalayan marmots from three regions of Qinghai Province, China. B, Schematic diagram of the genome organization of HMBOV1 and HMBOV2.

\section{Genomic characterization and analyses of HMBOV1 and HMBOV2}

The nearly complete genomes of HMBOV1 and HMBOV2 were 4,671 and 4,887 nucleotide in length, with a $\mathrm{G}+\mathrm{C}$ content of $39.5 \%$ and $45.6 \%$, respectively (Figure 1B). Putative ORFs of two novel viruses were predicted using NCBI's ORF Finder. Similar to other BOVs, the HMBOV1 and HMBOV2 genomes contained three ORFs: ORF1, encoding the partial nonstructural protein NS1 of 583 aa in HMBOV1 and full NS1 of 794 aa in HMBOV2; ORF2, encoding two overlapping structural proteins VP1/VP2 (617/629 aa and 550/498 aa respectively, differing by 67/131 amino acids at the N-terminus of VP1); and ORF3, encoding a highly phosphorylated nonstructural protein NP1 (246/200 aa). ORF3 is a unique feature in the middle of the BOV genome. NP1 of HMBOV2 shared a short overlapping sequence with the NP1 and NS1/VP1 genes, as also seen in human BOVs. By contrast, the NP1 and NS1/VP1 genes of HMBOV1 were separated, similar to PBoV-like viruses. Conserved motifs associated with rolling circle replication, helicase, and ATPase were also identified in ORF1 of HMBOV1 and HMBOV2. The conserved motifs of the $\mathrm{Ca}^{2+}$ binding loop (YLGPF) and the catalytic center (HDXXY) of phospholipase A2, required for parvovirus infectivity and located within the VP1-unique (VP1u) region (Zádori et al., 2001), were identified in VP1 of HMBOV2 but, as in PBoVlike viruses, not in VP1 of HMBOV1 (Figure 1B). The GenBank accession numbers for the sequences of HMBOV1 and HMBOV2 are MF464268 and MF464269, respectively.

Genomic analysis using BLASTN showed that HMBOV1 shared the highest identity of $76.0 \%$ with the complete genome of porcine BOV-SX (HQ223038) (Zeng et al., 2011). The NS1, NP1, and VP1/VP2 regions of HMBOV1 also had the highest amino acid similarity $(73.9 \%, 53.0 \%$, and $78.0 \%$ ) with those of porcine BOV-SX, compared with all BOV sequences. The three regions in HMBOV2 showed the highest similarity to those of porcine BOV 1-2 (Cheng et al., 2010), California sea lion BOV $1-4$, and bat BOVs, with amino acid identities of $45.2 \%-45.7 \%, 47.1 \%-48.6 \%$, $48.9 \%, 47.8 \%-50.4 \%, 50.7 \%-51.2 \%, 47.8 \%$, and $54.8 \%-$ $55.1 \%, 52.0 \%-53.3 \%, 52.0 \%$, respectively. HMBOV1 and HMBOV2 differed in their genomic sequences, with $40.6 \%$, $36.3 \%$, and $42.1 \%$ amino acid identities in their NS1, NP1, and VP1 regions, respectively. The identities between HMBOV1 and HMBOV2 and other BOVs are shown in 
detail in Table 1.

\section{Phylogenetic analysis}

Phylogenetic trees of the proteins NS1, NP1, and VP1 in HMBOV1 and HMBOV2 and other members of the genus Bocaparvovirus were constructed from the respective amino acid sequences. In the NS1 region, HMBOV1 formed an independent branch related to PBOV-SX (Zeng et al., 2011), whereas HMBOV2 formed a distinct monophyletic tree and was most closely related to PBOV1-2 (Cheng et al., 2010) (Figure 2A). In the NP1 region, phylogenetic analysis showed that HMBOV1 also clustered tightly with PBOV$\mathrm{SX}$, while HMBOV2 displayed a distinct branch and was also most closely related to PBOV1-2 (Figure 2B). In the VP1 region, HMBOV1 also formed a monophyletic tree and tightly clustered with PBOV-SX, whereas HMBOV2 formed a single and separate monophyletic branch, although it was closely related to the clade that included HMBOV1 and PBOV-SX 2 (Figure 2C).

\section{DISCUSSION}

The identification of novel viruses in wild animals is a very important step in predicting potential threats to human and animal health (Kahn, 2006). Himalayan marmot is one of the most economically valuable animal for human beings. Thus, whether they harbor viruses that may readily spread to humans should be determined. A prerequisite is to obtain baseline viral epidemiological information on Himalayan marmots, as performed in this study. BOVs have been shown to associate with respiratory, enteric, and neurological dis- ease in humans and animals (Manteufel and Truyen, 2008; Jartti et al., 2012; Yu et al., 2013; Mori et al., 2013), severely influencing the health of humans and animals. Despite the diversity of BOVs in multiple mammalian species, studies in wild marmots are lacking. Here, we report two novel BOVs identified, by viral metagenomic analysis of the livers and feces of wild Marmota himalayana in the Qinghai-Tibetan Plateau, China. This is the first study to identify and report the nearly full-length genome sequences of Bocaparvovirus in marmots.

The ICTV currently defines new BOV species as those sharing $<85.0 \%$ aa identity in the NS1 gene with other species. A sequence comparison revealed that HMBOV1 and HMBOV2 were the most related to BOVs of porcine origin (PBOV-SX/1-2). In the NS1 region, HMBOV1 and HMBOV2 shared $<74.0 \%$ and $50.0 \%$ aa identity with other BOVs. Phylogenetic analyses of the NS1, NP1, and VP1 proteins showed that HMBOV1 and HMBOV2 formed two distinct monophyletic trees in the genus of Bocaparvovirus. The two novel viruses displayed a genomic organization typical of BOV (Manteufel and Truyen, 2008), with two main ORFs encoding NS1 and VP1/VP2 and a third specific ORF (ORF3) encoding NP1, a highly phosphorylated protein that is essential for viral replication. Based on these findings, HMBOV1 and HMBOV2 were classified as two distinct new species (tentatively named as Himalayan marmot bocaparvovirus 1,2) of the Bocaparvovirus genus. This finding expands our current knowledge of the diversity of BOVs and their hosts.

Although the prevalence of HMBOV1 in 300 liver samples from Himalayan marmots was relatively low, five HMBOV1-positive liver samples were collected in the same region (a total of 60 liver samples collected), suggesting

Table 1 Amino acid identity (\%) between HMBOV1 and HMBOV2 and other bocaparvoviruses ${ }^{\mathrm{a}}$

\begin{tabular}{|c|c|c|c|c|c|c|}
\hline & \multicolumn{3}{|c|}{ HMBOV1 } & \multicolumn{3}{|c|}{ HMBOV2 } \\
\hline & NS1 & NP1 & VP1 & NS1 & NP1 & VP1 \\
\hline HMBOV2 & $73.9 \%$ & $53.0 \%$ & $78.0 \%$ & & & \\
\hline HBOV1-4 & $26.0 \%-35.4 \%$ & $29.4 \%-33.1 \%$ & $33.6 \%-35.1 \%$ & $34.4 \%-35.2 \%$ & $40.5 \%-44.1 \%$ & $43.2 \%-45.7 \%$ \\
\hline PBOV1-2 & $42.5 \%$ & $34.6 \%-35.4 \%$ & $40.5 \%-42.0 \%$ & $45.2 \%-45.7 \%$ & $47.8 \%-50.4 \%$ & $54.8 \%-55.1 \%$ \\
\hline PBOV3-4 & $38.3 \%-38.5 \%$ & $26.0 \%-28.5 \%$ & $33.2 \%-34.3 \%$ & $34.1 \%-35.3 \%$ & $33.8 \%-34.7 \%$ & $43.5 \%-43.6 \%$ \\
\hline PBOV-SX & $73.9 \%$ & $53.0 \%$ & $78.0 \%$ & $40.0 \%$ & $38.3 \%$ & $40.0 \%$ \\
\hline GBOV & $29.4 \%-34.7 \%$ & $29.4 \%-30.2 \%$ & $25.7 \%-33.8 \%$ & $34.0 \%-37.8 \%$ & $40.9 \%-42.0 \%$ & $42.6 \%-43.5 \%$ \\
\hline RBOV & $34.3 \%$ & $26.9 \%$ & $37.6 \%$ & $36.5 \%$ & $32.6 \%$ & $49.0 \%$ \\
\hline CSLBOV1-4 & $34.7 \%-35.3 \%$ & $29.3 \%-32.1 \%$ & $38.4 \%-39.4 \%$ & $47.1 \%-48.6 \%$ & $50.7 \%-51.2 \%$ & $52.0 \%-53.3 \%$ \\
\hline FeBOV & $34.2 \%$ & $28.2 \%$ & $39.2 \%$ & $43.6 \%$ & $38.2 \%$ & $51.9 \%$ \\
\hline $\mathrm{CaBOV} / \mathrm{MVC}$ & $37.6 \%-37.9 \%$ & $31.2 \%-31.6 \%$ & $41.8 \%-42.7 \%$ & $45.0 \%-46.6 \%$ & $45.1 \%$ & $53.1 \%-53.7 \%$ \\
\hline BoBOV & $35.3 \%$ & $34.7 \%$ & $33.8 \%$ & $32.3 \%$ & $44.1 \%$ & $15.8 \%$ \\
\hline BaBOV & $39.1 \%$ & $27.5 \%$ & $40.7 \%$ & $48.9 \%$ & $47.8 \%$ & $52.0 \%$ \\
\hline
\end{tabular}

a) HBOV, human bocaparvovirus; PBOV, porcine bocaparvovirus; GBOV, gorilla bocaparvovirus; RBOV, rabbit bocaparvovirus; CSLBOV, California sea lion bocaparvovirus; FeBOV, feline bocaparvovirus; CaBOV, canine bocaparvovirus; MVC, minute virus of canines; BoBOV, bovine bocaparvovirus; BaBOV, bat bocaparvovirus. 


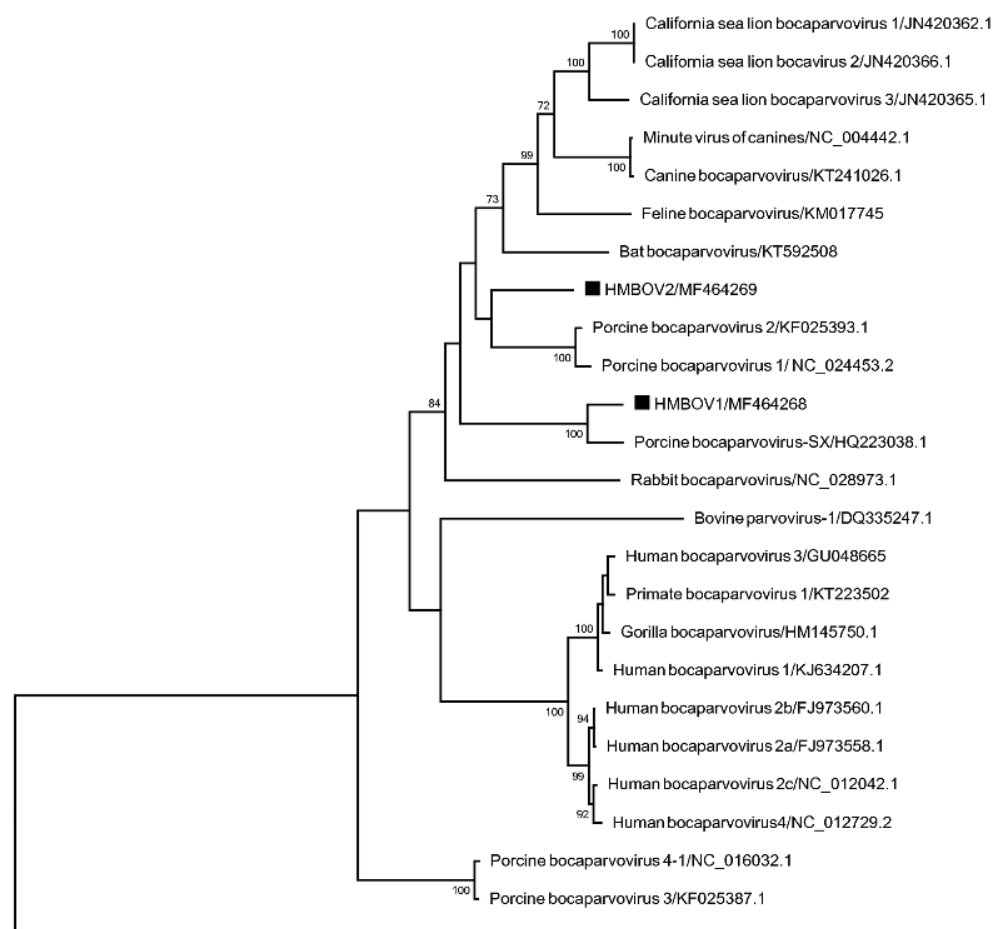

Canine parvovirus/EF011664.1 


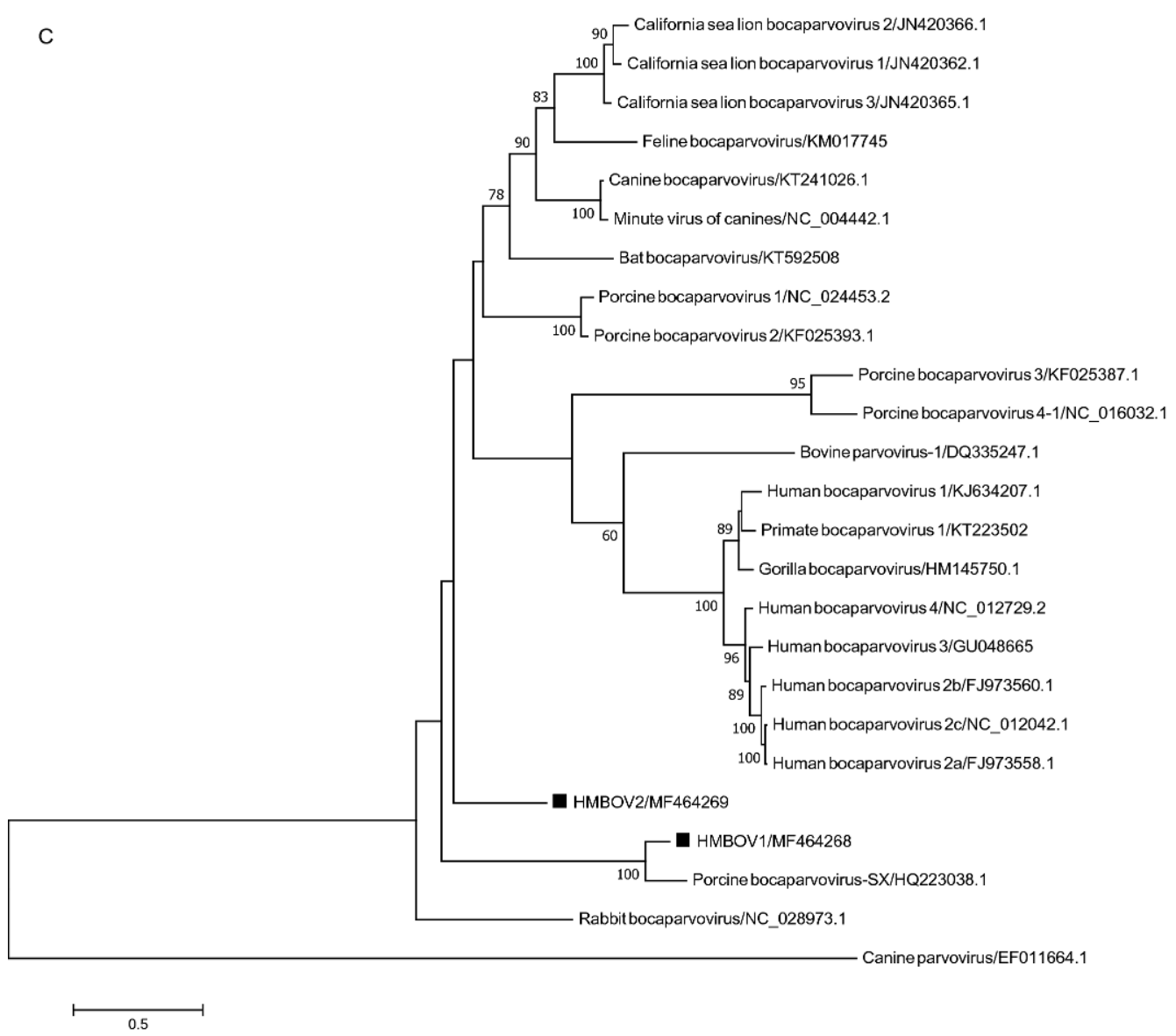

Figure 2 Phylogenetic tree based on the amino acid sequences of NS1 (A), NP1 (B), and VP1 (C) of HMBOV1 and HMBOV2 and of other bocaparvoviruses. The trees were constructed by the Maximum likelihood method with datasets of 1,000 replicates through the MEGA 6.0 software. The position of HMBOV1 and HMBOV2 are indicated by

HMBOV1 may be only prevalent in the local region. Additionally, the $100.0 \%$ nt identity of all the partial NS1 sequences suggested that HMBOV1 has been stably circulating in the local Himalayan marmot population. HMBOV2 was detected in approximately $5.5 \%$ of the stool samples from these animals and was thus of a relatively low prevalence. HMBOV1 was not detected in the stool samples and HMBOV2 not in the liver samples, suggesting that different species of BOV vary in their tissue tropism. Phylogenetic analysis of the NS1, NP1, and VP1 proteins consistently showed that HMBOV1 was most closely related to PBOVSX $(73.9 \%, 53.0 \%$, and $78.0 \%$ aa identities in NS1, NP1, and VP1, respectively), identified in serum samples from pigs in China (Zeng et al., 2011). This result implies that both HMBOV1 and PBOV-SX share an HMBOV1-like common ancestor and the possibility of cross-species transmission between marmots and pigs for BOVs. As for HMBOV2, the phylogenetic analysis showed that it formed a mostly separated and monophyletic branch among the bocaparvoviruses. Based on their NS1, NP1, and VP1 genes, HMBOV1 and HMBOV2 were all genetically more similar to porcine bocaparvoviruses PBOV-SX, respectively, and thus unlikely to have derived from inter-species recombinant viruses. In addition, the relatively far phylogenetic distance between HMBOV1 and HMBOV2 suggests that additional novel viral species may be present in marmots. Overall, this data will improve our understanding of the evolution and ecology of BOVs.

BOVs are mostly associated with respiratory and enteric diseases, especially in young children and animals (Manteufel and Truyen, 2008; Jartti et al., 2012), but they were recently implicated in encephalitis both in children and adults (Yu et al., 2013; Mori et al., 2013). A BOV was also identified in the canine liver, with infection associated with severe hemorrhagic gastroenteritis, necrotizing vasculitis, granulomatous lymphadenitis, and anuric renal failure (Li et al., 2013). These data suggest multiple organs could be localized by BOVs. In this study, HMBOV1 was identified in the liver of Himalayan marmots, leading us to ask whether HMBOV1 could really infect the liver and cause similar illness seen in canine. Thus, further clinical studies addressing in vivo infection with HMBOV1/2 in marmots will be necessary to test Koch's postulates and study its pathogenesis. However, in this study, there is a limitation that the fecal 
and liver samples, or other tissue samples from the same Himalayan marmots were not collected. As a result, the present study could not address the issue whether HMBOV1/ 2 are located simultaneously in other organs of Himalayan marmots.

Previous reports have described the capacity of parvoviruses to infect new host species (Hoelzer and Parrish, 2010; Parrish and Kawaoka, 2005). Although there is as yet no report of the cross-species transmission of BOVs from animals to humans, the mutation rate of BOVs as singlestranded DNA viruses approaches that of RNA viruses (Shackelton et al., 2005; Hoelzer et al., 2008). Given the increasing contact between humans and Himalayan marmots, studies on the potential of HMBOV1 and HMBOV2 to infect humans are warranted. These should include epidemiological studies that make use of serological assays of local human inhabitants to evaluate their risk of infection. In addition, as recent ecological and environmental changes alter the relationships between wild animals and humans (Tompkins et al., 2015), additional, similar viral discovery studies in wild animals should be conducted to prevent and control emerging viral diseases in humans.

\section{MATERIALS AND METHODS}

\section{Specimens}

Three hundred liver samples were collected from 300 wild Himalayan marmots and 99 fecal specimens from 99 other wild Himalayan marmots from three different regions (Dulan, Wulan, Tianjun counties) of the Qinghai-Tibetan Plateau, Qinghai Province, China, from 2013 to 2014. All of the samples were transported to our laboratory on dry ice and frozen at $-80^{\circ} \mathrm{C}$, until further processing.

\section{High-throughput sequencing}

The liver and fecal samples were diluted in phosphate-buffered saline $(1: 10 \mathrm{w} / \mathrm{v})$, homogenized/vortexed, and centrifuged at $8,000 \times g$ for $10 \mathrm{~min}$. Every 10 samples were pooled. After filtration of the viral suspension through 0.45 and $0.22-\mu \mathrm{m}$ filters, the filtrate was treated with DNase (Turbo DNase, Ambion, USA) to digest unprotected nucleic acids. Total nucleic acids were extracted using the QIAamp viral mini kit (Qiagen, Hilden, Germany) according to the manufacturer's protocol. Viral nucleic acid libraries were constructed by sequence-independent random reverse transcription PCR using the primer K-8N (5'-GTTCCCAGTCACGATANNNNNNNN-3') and sequenced using the Illumina Miseq platform with 250-base paired ends (Illumina, San Diego, CA, USA). The sequencing data were further analyzed by the customized informatics pipeline Virus Hunter, as previously described (Finkbeiner et al.,
2008).

\section{Detection of HMBOV1 and HMBOV2}

To screen HMBOV1 and HMBOV2 in the liver and stool of Himalayan marmots, cDNAs were generated from the 300 liver tissues and 99 fecal samples using random primers and SuperScript III reverse transcriptase (Invitrogen, Carlsbad, CA, USA). A 386 base pair (bp) fragment was then amplified using hemi-nested PCR, targeting a portion of the NS1 gene of HMBOV1 and HMBOV2, and the primers MBOVF1 (5'ATGCATGYTGACTGGGTRGAAC-3'), MBOVF1 (5'TGYTGACTGGGTRGAACCAGC-3'), and MBOVR (5'AGACGAAGTCCTGTGAATGAG-3'). The two rounds of amplification were achieved under the following conditions: $94^{\circ} \mathrm{C}$ for $5 \mathrm{~min}$, followed by 35 cycles $\left(94^{\circ} \mathrm{C}\right.$ for $30 \mathrm{~s}, 53^{\circ} \mathrm{C}$ for $30 \mathrm{~s}$, and $72^{\circ} \mathrm{C}$ for $1 \mathrm{~min}$ ) and then $72^{\circ} \mathrm{C}$ for $5 \mathrm{~min}$. The PCR amplicons were electrophoresed and purified on a $1.5 \%$ agarose gel. The positive samples were then sequenced using the Big-Dye terminator cycle sequencing kit and the ABI Prism 310 genetic analyzer (Applied Biosystems, Foster City, CA, USA).

\section{Genomic sequencing}

To obtain the complete sequences of HMBOV1 and HMBOV2, a routine PCR and genome-walking kit (Takara Bio Inc., Japan) was used to amplify the unknown sequences. The specific primers employed were based on the contigs obtained by high-throughput sequencing and using the newly amplified sequences. To confirm the final genomic sequence of HMBOV1 and HMBOV2, overlapping PCR and LA-Taq polymerase (Takara Bio Inc.) were used to amplify three long fragments.

\section{Genomic analysis}

Full-length sequences were assembled using SeqMan software (DNASTAR, Inc., Madison, WI, USA). The ORFs for HMBOV1 and HMBOV2 were identified using the ORF finder of the National Center for Biotechnology Information (NCBI; http://www.ncbi.nlm.nih.gov/gorf/gorf.html). Multiple nucleotide and deduced amino acid sequence alignments were analyzed using Clustalx software (version 1.83). Pairwise nucleotide (nt) and amino acid (aa) identities between novel BOVs and other parvoviruses were determined, using DNAMAN software.

\section{Phylogenetic analysis}

To determine the phylogenetic relationship of HMBOV1 and HMBOV2, the amino acid sequences of the NS1, NP1, and VP1 proteins of HMBOV1 and HMBOV2 and other par- 
voviruses were aligned using Clustalx (version 1.83). The Maximum likelihood (ML) phylogenetic trees of them were constructed with the aa substitution model of Le Gascule_2008 model with Freqs and gamma $(\mathrm{LG}+\mathrm{G}+\mathrm{F})$ by the MEGA 6.0 software.

Compliance and ethics The author(s) declare that they have no conflict of interest. The study was approved by the Chinese CDC's Ethics Committee on the use of animals and complied with Chinese ethics laws and regulations.

Acknowledgements We thank the department of W. Ian Lipkin from Center for Infection and Immunity, Columbia University, for the help of bioinformatics analysis. This work was funded by the Special National Project on Research and Development of Key Biosafety Technologies (2016YFC1201900) and the National Natural Science Foundation of China (81290345).

Abixanti, F.R., and Warfield, M.S. (1961). Recovery of a hemadsorbing virus (HADEN) from the gastrointestinal tract of calves. Virology 14, 288-289.

Ao, Y.Y., Yu, J.M., Li, L.L., Cao, J.Y., Deng, H.Y., Xin, Y.Y., Liu, M.M., Lin, L., Lu, S., Xu, J.G., and Duan, Z.J. (2017). Diverse novel astroviruses identified in wild Himalayan marmots. J Gen Virol 98, 612-623.

Balkhy, H.H., Sabella, C., and Goldfarb, J. (1998). Parvovirus: a review. Bull Rheum Dis 47, 4-9.

Binn, L.N., Lazar, E.C., Eddy, G.A., and Kajima, M. (1970). Recovery and characterization of a minute virus of canines. Infect Immun 1, 503-508.

Cashman, O., and O'Shea, H. (2012). Detection of human bocaviruses 1, 2 and 3 in Irish children presenting with gastroenteritis. Arch Virol 157, 1767-1773.

Cheng, W., Li, J., Huang, C., Yao, D., Liu, N., Cui, S., Jin, Y., and Duan, Z. (2010). Identification and nearly full-length genome characterization of novel porcine bocaviruses. PLoS ONE 5, e13583.

Christianson, W.T. (1992). Stillbirths, mummies, abortions, and early embryonic death. Vet Clin North Am Food Anim Pract 8, 623-639.

Cságola, A., Lőrincz, M., Cadar, D., Tombácz, K., Biksi, I., and Tuboly, T. (2012). Detection, prevalence and analysis of emerging porcine parvovirus infections. Arch Virol 157, 1003-1010.

Drosten, C., Günther, S., Preiser, W., van der Werf, S., Brodt, H.R., Becker, S., Rabenau, H., Panning, M., Kolesnikova, L., Fouchier, R.A.M., Berger, A., Burguière, A.M., Cinatl, J., Eickmann, M., Escriou, N., Grywna, K., Kramme, S., Manuguerra, J.C., Müller, S., Rickerts, V., Stürmer, M., Vieth, S., Klenk, H.D., Osterhaus, A.D.M.E., Schmitz, H., and Doerr, H.W. (2003). Identification of a novel coronavirus in patients with severe acute respiratory syndrome. N Engl J Med 348, 1967-1976.

Finkbeiner, S.R., Allred, A.F., Tarr, P.I., Klein, E.J., Kirkwood, C.D., and Wang, D. (2008). Metagenomic analysis of human diarrhea: viral detection and discovery. PLoS Pathog 4, e1000011.

Gire, S.K., Goba, A., Andersen, K.G., Sealfon, R.S.G., Park, D.J., Kanneh, L., Jalloh, S., Momoh, M., Fullah, M., Dudas, G., Wohl, S., Moses, L. M., Yozwiak, N.L., Winnicki, S., Matranga, C.B., Malboeuf, C.M., Qu, J., Gladden, A.D., Schaffner, S.F., Yang, X., Jiang, P.P., Nekoui, M., Colubri, A., Coomber, M.R., Fonnie, M., Moigboi, A., Gbakie, M., Kamara, F.K., Tucker, V., Konuwa, E., Saffa, S., Sellu, J., Jalloh, A.A., Kovoma, A., Koninga, J., Mustapha, I., Kargbo, K., Foday, M., Yillah, M., Kanneh, F., Robert, W., Massally, J.L.B., Chapman, S.B., Bochicchio, J., Murphy, C., Nusbaum, C., Young, S., Birren, B.W., Grant, D.S., Scheiffelin, J.S., Lander, E.S., Happi, C., Gevao, S.M., Gnirke, A., Rambaut, A., Garry, R.F., Khan, S.H., and Sabeti, P.C. (2014). Genomic surveillance elucidates Ebola virus origin and transmission during the 2014 outbreak. Science 345, 1369-1372.

Gunn, L., Collins, P.J., Fanning, S., McKillen, J., Morgan, J., Staines, A., and O'Shea, H. (2015). Detection and characterisation of novel boca- virus (genusBocaparvovirus) and gastroenteritis viruses from asymptomatic pigs in Ireland. Infect Ecol Epidemiol 5, 27270.

Haagmans, B.L., Al Dhahiry, S.H.S., Reusken, C.B.E.M., Raj, V.S., Galiano, M., Myers, R., Godeke, G.J., Jonges, M., Farag, E., Diab, A., Ghobashy, H., Alhajri, F., Al-Thani, M., Al-Marri, S.A., Al Romaihi, H. E., Al Khal, A., Bermingham, A., Osterhaus, A.D.M.E., AlHajri, M.M., and Koopmans, M.P.G. (2014). Middle East respiratory syndrome coronavirus in dromedary camels: an outbreak investigation. Lancet Infect Dis $14,140-145$.

He, B., Li, Z., Yang, F., Zheng, J., Feng, Y., Guo, H., Li, Y., Wang, Y., Su, N., Zhang, F., Fan, Q., and Tu, C. (2013). Virome profiling of bats from Myanmar by metagenomic analysis of tissue samples reveals more $\mathrm{n}$ ovel mammalian viruses. PLoS ONE 8, e61950.

Hoelzer, K., Shackelton, L.A., Parrish, C.R., and Holmes, E.C. (2008). Phylogenetic analysis reveals the emergence, evolution and dispersal of carnivore parvoviruses. J Gen Virol 89, 2280-2289.

Hoelzer, K., and Parrish, C.R. (2010). The emergence of parvoviruses of carnivores. Vet Res 41, 39.

Jartti, T., Hedman, K., Jartti, L., Ruuskanen, O., Allander, T., and Söderlund-Venermo, M. (2012). Human bocavirus- the first 5 years. Rev Med Virol 22, 46-64.

Jones, K.E., Patel, N.G., Levy, M.A., Storeygard, A., Balk, D., Gittleman, J.L., and Daszak, P. (2008). Global trends in emerging infectious diseases. Nature 451, 990-993.

Jula, A., Waris, M., Kantola, K., Peltola, V., Söderlund-Venermo, M., Hedman, K., and Ruuskanen, O. (2013). Primary and secondary human bocavirus 1 infections in a family, Finland. Emerg Infect Dis 19, 13281331.

Kahn, L.H. (2006). Confronting zoonoses, linking human and veterinary medicine. Emerg Infect Dis 12, 556-561.

Kapoor, A., Slikas, E., Simmonds, P., Chieochansin, T., Naeem, A., Shaukat, S., Alam, M.M., Sharif, S., Angez, M., Zaidi, S., and Delwart, E. (2009). A newly identified bocavirus species in human stool. J Infect Dis 199, 196-200.

Kapoor, A., Mehta, N., Esper, F., Poljsak-Prijatelj, M., Quan, P.L., Qaisar, N., Delwart, E., and Lipkin, W.I. (2010). Identification and characterization of a new bocavirus species in gorillas. PLoS ONE 5, e11948.

Lanave, G., Martella, V., Farkas, S.L., Marton, S., Fehér, E., Bodnar, L., Lavazza, A., Decaro, N., Buonavoglia, C., and Bányai, K. (2015). Novel bocaparvoviruses in rabbits. Veterinary J 206, 131-135.

Lau, S.K.P., Woo, P.C.Y., Yeung, H.C., Teng, J.L.L., Wu, Y., Bai, R., Fan, R.Y.Y., Chan, K.H., and Yuen, K.Y. (2012). Identification and characterization of bocaviruses in cats and dogs reveals a novel feline bocavirus and a novel genetic group of canine bocavirus. J Gen Virol 93, $1573-1582$.

Li, L., Pesavento, P.A., Leutenegger, C.M., Estrada, M., Coffey, L.L., Naccache, S.N., Samayoa, E., Chiu, C., Qiu, J., Wang, C., Deng, X., and Delwart, E. (2013). A novel bocavirus in canine liver. Virol J 10, 54.

Li, L., Shan, T., Wang, C., Cote, C., Kolman, J., Onions, D., Gulland, F.M. D., and Delwart, E. (2011). The fecal viral flora of California sea lions. J Virol 85, 9909-9917.

Manteufel, J., and Truyen, U. (2008). Animal bocaviruses: a brief review. Intervirology 51, 328-334.

Meng, X.J. (2012). Emerging and re-emerging swine viruses. Transbound Emerg Dis 59, 85-102.

Mori, D., Ranawaka, U., Yamada, K., Rajindrajith, S., Miya, K., Perera, H. K.K., Matsumoto, T., Dassanayake, M., Mitui, M.T., Mori, H., Nishizono, A., Söderlund-Venermo, M., and Ahmed, K. (2013). Human Bocavirus in Patients with Encephalitis, Sri Lanka, 2009-2010. Emerg Infect Dis 19, 1859-1862.

Parrish, C.R., and Kawaoka, Y. (2005). The origins of new pandemic viruses: the acquisition of new host ranges by canine parvovirus and influenza A viruses. Annu Rev Microbiol 59, 553-586.

Rotzén-Östlund, M., Eriksson, M., Tiveljung Lindell, A., Allander, T., Zweygberg Wirgart, B., and Grillne, L. (2014). Children with multiple viral respiratory infections are older than those with single viruses. Acta Paediatr Int J Paediatr 103, 100-104. 
Shackelton, L.A., Parrish, C.R., Truyen, U., and Holmes, E.C. (2005). High rate of viral evolution associated with the emergence of carnivore parvovirus. Proc Natl Acad Sci USA 102, 379-384.

Tompkins, D.M., Carver, S., Jones, M.E., Krkošek, M., and Skerratt, L.F. (2015). Emerging infectious diseases of wildlife: a critical perspective. Trends Parasitol 31, 149-159.

Yang, S., Wang, Y., Li, W., Fan, Z., Jiang, L., Lin, Y., Fu, X., Shen, Q., Sun, Z., Wang, X., Deng, X., Zhang, W., and Delwart, E. (2016). A novel bocavirus from domestic mink, China. Virus Genes 52, 887-890.

Yu, J.M., Chen, Q.Q., Hao, Y.X., Yu, T., Zeng, S.Z., Wu, X.B., Zhang, B., and Duan, Z.J. (2013). Identification of human bocaviruses in the cerebrospinal fluid of children hospitalized with encephalitis in China. J Clin Virol 57, 374-377.

Yu, J.M., Li, L.L., Zhang, C.Y., Lu, S., Ao, Y.Y., Gao, H.C., Xie, Z.P., Xie,
G.C., Sun, X.M., Pang, L.L., Xu, J.G., Lipkin, W.I., and Duan, Z.J. (2016). A novel hepatovirus identified in wild woodchuck Marmota himalayana. Sci Rep 6, 22361.

Zádori, Z., Szelei, J., Lacoste, M.C., Li, Y., Gariépy, S., Raymond, P., Allaire, M., Nabi, I.R., and Tijssen, P. (2001). A viral phospholipase A2 is required for parvovirus infectivity. Dev Cell 1, 291-302.

Zeng, S., Wang, D., Fang, L., Ma, J., Song, T., Zhang, R., Chen, H., and Xiao, S. (2011). Complete coding sequences and phylogenetic analysis of porcine bocavirus. J Gen Virol 92, 784-788.

Zhai, S., Yue, C., Wei, Z., Long, J., Ran, D., Lin, T., Deng, Y., Huang, L., Sun, L., Zheng, H., Gao, F., Zheng, H., Chen, S., and Yuan, S. (2010). High prevalence of a novel porcine bocavirus in weanling piglets with respiratory tract symptoms in China. Arch Virol 155, 1313-1317. 